\title{
Sensitization to Airborne Ascospores, Basidiospores, and Fungal Fragments in Allergic Rhinitis and Asthmatic Subjects in San Juan, Puerto Rico
}

\author{
Félix E. Rivera-Mariani $^{a} \quad$ Sylvette Nazario-Jiménez ${ }^{b} \quad$ Fernando López-Malpica $^{b}$ \\ Benjamín Bolaños-Rosero ${ }^{\mathrm{a}}$ \\ Departments of a Microbiology and ${ }^{b}$ Rheumatology and Immunology, School of Medicine, University of \\ Puerto Rico - Medical Sciences Campus, San Juan, Puerto Rico
}

\section{Key Words}

Airborne allergens $\cdot$ Ascospores $\cdot$ Asthma $\cdot$ Fungi .

Basidiospores $\cdot$ Particulates $\cdot$ Allergic rhinitis $\cdot$ Sensitization

\begin{abstract}
Background: Fungal spores are the predominant biological particulate in the atmosphere of Puerto Rico, yet their potential as allergens has not been studied in subjects with respiratory allergies. The purpose of this study was to determine the level of sensitization of subjects with respiratory allergies to these particles. Methods: Serum samples were drawn from 33 subjects with asthma, allergic rhinitis, or nonallergic rhinitis and 2 controls with different skin prick test reactivity. An MK-3 sampler was used to collect air samples and the reactivity of the sera to fungal particles was detected with a halogen immunoassay. Results: All subjects reacted to at least 1 fungal particle. Thirty-one subjects reacted to ascospores, 29 to basidiospores, 19 to hyphae/fungal fragments, and 12 to mitospores. The median percentage of haloes in allergic rhinitis subjects was $4.82 \%$ while asthma or nonallergic rhinitis subjects had values of 1.09 and $0.39 \%$, respectively. Subjects with skin prick tests positive to $3,2,1$, or no extract had 5.24, 1.09, 1.61, and, $0.57 \%$ of haloed particles,
\end{abstract}

respectively. If skin prick tests were positive to basidiomycetes, pollen, animals, or deuteromycetes, the percentages of haloes were $4.72,4.15,3.63$, and 3.31\%, respectively. Of all haloed particles, $46 \%$ were unidentified, $25 \%$ ascospores, $20 \%$ basidiospores, $7 \%$ hyphae/fungal fragments, and $2 \%$ mitospores. IgE levels and the number of positive skin prick test extracts correlated with the percentage of haloes. Conclusion: In tropical environments, sensitization to airborne basidiomycetes, ascomycetes, and fungal fragments seems to be more prevalent than sensitization to mitospores in subjects with active allergies, suggesting a possible role in exacerbations of respiratory allergies.

Copyright $\odot 2011$ S. Karger AG, Basel

\section{Introduction}

Allergic respiratory diseases have been increasing worldwide [1,2]. According to the World Health Organization [3], the United States is among the countries with the highest mortality rates due to asthma, and Puerto Rico has one of the highest incidences among the 50 states and its territories [4-10]. Although many studies suggest that a genetic predisposition is the most impor-

\section{KARGER}

๑ 2011 S. Karger AG, Basel

Fax +41613061234 E-Mail karger@karger.ch www.karger.com www.karger.com/iaa
Correspondence to: Dr. Benjamín Bolaños-Rosero

Department of Microbiology, School of Medicine, UPR-MSC

PO Box 365067

San Juan 00936-5067 (Puerto Rico)

Tel. +1 787759 8228, Fax +1 787756 8475, E-Mail benjamin.bolanos@upr.edu 
tant factor $[2,11,12]$, others propose a significant role of environmental variables including outdoor airborne allergens $[9,10,13]$. For this reason, it is important to directly study the role of aeroallergens in inducing episodes of rhinitis and asthma.

The predominant biological airborne particulates in many temperate and tropical regions are pollen and fungal spores [14-16], and some studies have revealed that fungal fragments are also prevalent [17-21]. Furthermore, a significant number of species of pollen and fungi have been described as allergens or as having the potential to be allergenic [22-24]. Nevertheless, in many geographic locations fungal spores are the principal biological component in the atmosphere and various studies have identified their importance in episodes of respiratory allergic diseases [22, 23, 25-28]. Therefore, it is reasonable to expect that approximately $10 \%$ of the population could be allergic to airborne molds. In a recently published study, we characterized the biological airborne particulates in the atmosphere of San Juan, Puerto Rico (SJ) [14], in which basidiospores and ascospores represented over $90 \%$ of the outdoor biological particulates and fungal spores throughout the year. In addition, there was an intradiurnal variation directly affected by the high humidity and dew point but inversely affected by wind and wind gusts. This is not the case in temperate regions, in which mitospores (deuteromycetes or Fungi Imperfecti) such as Cladosporium and Alternaria are the most common. Numerous studies have described the allergenic potential of these spores in temperate areas [16, 29-31], but very few have demonstrated the same potential with basidiospores and ascospores, or directly with airborne fungal spores, due to the difficulty of preparing extracts $[24-26,32,33]$.

Sensitization to a specific allergen can be detected in vivo via the skin prick test (SPT) or in vitro (e.g. radioallergosorbent test) by determining the presence and levels of immunoglobulin E (IgE) when the serum of a patient is challenged with an extract $[1,34]$. One important limitation of these tests is that they are dependent on the extract's quality which is known to be affected by many variables including intraspecies mutations, batch-tobatch variation, and culture conditions $[24,34,35]$. In addition, extracts are not available for many fungi or are very difficult to prepare, which could lead to a subdiagnosis of fungal allergies. Testing a whole-air sample extract would not be specific because of the heterogeneity of biological airborne particulates, and the presence of endotoxin may lead to secondary reactions.

The halogen immunoassay, initially developed by O'Meara et al. [36] to detect inhaled cat allergen, de- scribed in detail by Tovey et al. [37], and later refined for fungal allergens by Mitakakis et al. [38] and Green et al. $[19-21,35,39,40]$ is an immunoblotting method with some similarities to the enzyme-link immunosorbent assay and Western blot techniques. Advantages of this technique are that it is not dependent on any extract and the particle to be tested is induced to elute allergen. In addition, the assay is performed on a sealed protein binding membrane that captures the allergen-IgE complexes that can be observed under the microscope. It has been used to test various mitosporic fungi and nonfungal allergens (e.g. cat) and, more importantly, it can also be used to test against air samples collected with a volumetric air sampler.

Given the importance of airborne fungal spores in allergic respiratory diseases and their predominance in many geographical areas, the purpose of this study was to determine the sensitization of sera from subjects with asthma, allergic rhinitis, or nonallergic rhinitis to airborne fungal particulates present in the atmosphere. Because of our previous aerobiological study in SJ, our hypothesis was that the predominant airborne spores in our atmosphere (basidiospores and ascospores) have higher percentages of reactivity compared to mitosporic fungi.

\section{Materials and Methods}

\section{Recruitment of Volunteers}

With the approval of the Institutional Review Board (IRB) of the University of Puerto Rico, Medical Sciences Campus (UPRMSC), participants were selected from among staff and students of the UPR-MSC and patients seen by UPR-MSC faculty-physicians at external allergy clinics. Inclusion criteria for the study were a self-reported history of suffering from nasal allergies or asthma, having had a stable medical regimen for the previous 30 days, being noninstitutionalized, and being $>15$ years of age. Exclusion criteria were the current use of oral steroids for the treatment of asthma or being in an immunosuppresor regimen, any type of cancer within the last 6 months, infection of the upper respiratory tract within the last 2 weeks, being a smoker, having dementia or other conditions that would not allow the accurate self-reporting of data, having self-reported poor compliance with any medical therapy, being institutionalized, and being pregnant. The purpose of the study was explained to each individual in understandable terms and subjects were presented with an informed-consent form. Thirty-three participants consented to being part of the study. All of them were $>22$ years of age, with female volunteers being predominantly represented ( 30 females and 3 males). Allergic rhinitis was diagnosed based on the history of the current use of medications for rhinitis or nasal symptoms as determined by the nasal symptoms score and/or physician-diagnosed allergic rhinitis in addition to a positive skin test to relevant antigens based on the subject's history. Nonallergic rhinitis was diagnosed in the presence of current nasal symptoms, medication 
use, and a negative skin test. Negative controls were asymptomatic subjects who did not use any medications for rhinitis and who had negative skin test results. Asthma was diagnosed based on pulmonary symptoms of wheeze, cough, and shortness of breath, as well as on medication use and physician diagnosis. Chronic obstructive pulmonary disease (COPD) was diagnosed based on pulmonary symptoms and smoking history. When arranging subjects into groups based on the diagnosed disease, those who had allergic rhinitis and asthma were placed in the asthma group. The only subject with COPD was classified with asthmatics as this subject was also suffering from asthma.

\section{Human Sera}

Serum samples were drawn by trained medical technicians from 33 subjects (table 1) with no respiratory disease, asthma, or allergic or nonallergic rhinitis. SPT were performed using commercial and crude basidiospore extracts and the samples were stored at $-80^{\circ} \mathrm{C}$ until analysis. In the SPT, a wheal $3 \mathrm{~mm}$ larger than that of the negative control was considered positive. In addition, the IgE levels were determined at a reference laboratory using an Advia Centaur ${ }^{\circledR}$ CP Immunoassay System (Bayer Diagnostics, Greenburgh, N.Y., USA). In 4 of the subjects (with allergic rhinitis) the volume of sera was insufficient to determine both the IgE titer and the reactivity with a halogen immunoassay; for these participants, only the halogen immunoassay was used. The battery of SPT extracts included commercial extracts (Greer Laboratories, Lenoir, N.C., USA) of mitosporic fungi (Penicillium spp., Aerobasidium spp., Alternaria spp., Cladosporium spp., Acremonium spp., Fusarium spp., and Aspergillus spp.), 1 ascomycete (Chaetomium spp.), animals (dog, cat, mite, and cockroach), pollen (Bermuda, Johnson, Bahia, and trees), and crude extracts from basidiomycetes' spore prints (Ganoderma applanatum, Chlorophyllum molybdites, and Pleurotus ostreatus) which were prepared as previously described by Horner et al. [32].

\section{Collection of Air Samples}

An $80 \times 20 \mathrm{~mm}$ strip of ABgene PCR sealing film (Fisher Scientific, New Hampton, N.H., USA) was attached, with the adhesive surface facing upwards, to a microscope slide. The prepared slide was placed in an Allergenco ${ }^{\circledR}$ MK-3 sampler (Environmental Monitoring Systems, Inc., Charleston, S.C., USA) situated on the roof top of the main building of the UPR-RCM $(\sim 100 \mathrm{~m}$ above ground level) located in SJ. Outdoor air samples were collected at a volume of 15 liters $/ \mathrm{m}^{3}$ of air in March, May, June, and December of 2009 and in January of 2010. Air sampling was done only on days during these months when participants were recruited and sera were available for testing. The samples were collected from 4 p.m. to 8 a.m., which are the hours with the highest fungal concentration in the SJ atmosphere, at intervals of 10 min of sampling every 110 min of relapse. Although these hours may not reflect actual periods of individual exposure, we wanted to test as many fungal spores and particulates as possible. Sampling during daytime hours would have yielded very few spores or other nonbiological particulates. At 8 a.m. the slide was removed from the sampler and processed with the halogen immunoassay. In the afternoon (4 p.m.) of the same day another slide with adhesive tape was placed in the sampler. The same procedure was repeated on the days on which sera was drawn until enough air samples had been collected for all of the sera because on 1 adhesive film a limited number of mixed-cellulose ester protein binding membrane squares could be attached.
Table 1. Clinical data, IgE levels, and SPT results of the patients included in the study

\begin{tabular}{|c|c|c|c|c|}
\hline $\begin{array}{l}\mathrm{Pa}- \\
\text { tient }\end{array}$ & Condition & $\begin{array}{l}\text { Disease } \\
\text { code }\end{array}$ & $\begin{array}{l}\text { IgE } \\
(\mathrm{IU} / \mathrm{ml})\end{array}$ & $\mathrm{SPT}(+)$ \\
\hline 1 & NAR & 472 & ND & Gano, Chlor, grass \\
\hline 2 & AR, COPD & 477.8 & 107.1 & Mite, grass \\
\hline 3 & AR & 477.8 & ND & Mite, trees \\
\hline 4 & $\mathrm{AR}$, asthma & 493 & 117.8 & Mite, grass \\
\hline 5 & AR & 477.8 & 16.6 & Mite \\
\hline 6 & AR & 477.8 & ND & Gano, mite, cat \\
\hline 7 & AR & 477.8 & ND & Pen, mite, cockr, cat \\
\hline 8 & $\begin{array}{l}\text { AR, asthma, } \\
\text { conjunct }\end{array}$ & 493 & 49.6 & Mite, Gano \\
\hline 9 & Neg ctrl & control & 5.7 & None \\
\hline 10 & NAR & 472 & 4.9 & None \\
\hline 11 & Neg ctrl & control & 8.7 & Fusarium \\
\hline 12 & AR, asthma & 493 & 6.5 & Pleu, cockr \\
\hline 13 & NAR & 472 & 1 & None \\
\hline 14 & $\begin{array}{l}\text { Chronic } \\
\text { rhinitis }\end{array}$ & 472 & 29 & $\begin{array}{l}\text { Unable to be } \\
\text { determined }\end{array}$ \\
\hline 15 & NAR & 472 & 43.5 & Gano \\
\hline 16 & NAR & 472 & 1.5 & None \\
\hline 17 & AR & 477.8 & 338.8 & Pen \\
\hline 18 & AR, asthma & 493 & 27.5 & Mite \\
\hline 19 & NAR & 472 & 648.1 & None \\
\hline 20 & NAR, AD & 472 & 461.9 & None \\
\hline 21 & AR, asthma & 493 & 64.6 & Cockr \\
\hline 22 & NAR & 472 & 14.4 & None \\
\hline 23 & AR, asthma & 493 & 210.4 & Pleu, Gano \\
\hline 24 & $\mathrm{AR}$, asthma & 493 & 491.6 & Gano \\
\hline 25 & AR & 477.8 & 242.8 & Pen, mite \\
\hline 26 & NAR & 472 & 79.4 & None \\
\hline 27 & NAR & 472 & 12.3 & Gano, Chlor, Pleu, mite \\
\hline 28 & AR & 477.8 & 755.3 & Grass \\
\hline 29 & Asthma & 493 & 1,442 & $\begin{array}{l}\text { Mite, grass, Asp, Alt, } \\
\text { Gano, Chlor, mite }\end{array}$ \\
\hline 30 & AR & 477.8 & 307.7 & Grass \\
\hline 31 & AR & 477.8 & 100.5 & Grass \\
\hline 32 & AR & 477.8 & $2,017.1$ & $\begin{array}{l}\text { Asp, Alt, Gano, mite, } \\
\text { grass, Chlor, Pleu }\end{array}$ \\
\hline 33 & Asthma & 493 & $1,101.4$ & Grass \\
\hline
\end{tabular}

$\mathrm{AD}=$ Atopic dermatitis; $\mathrm{AR}=$ allergic rhinitis; conjunct $=$ conjunctivitis; $\mathrm{COPD}=$ chronic obstructive pulmonary disease; $\mathrm{NAR}=$ nonallergic rhinitis; neg ctrl = negative control; Alt $=A l$ ternaria spp.; Asp = Aspergillus spp; Chlor = Chlorophyllum molybdites; cockr = cockroach; Gano = Ganoderma applanatum; Pleu $=$ Pleurotus ostreatus .

\section{Halogen Immunoassay}

The patients' sera were blindly analyzed with the halogen immunoassay (fig. 1a) before the clinical, IgE level, and SPT data were provided. The assay was performed as described by Green et al. [39] but instead of the air sample being collected on the membrane it was collected on the adhesive tape as mentioned above. 




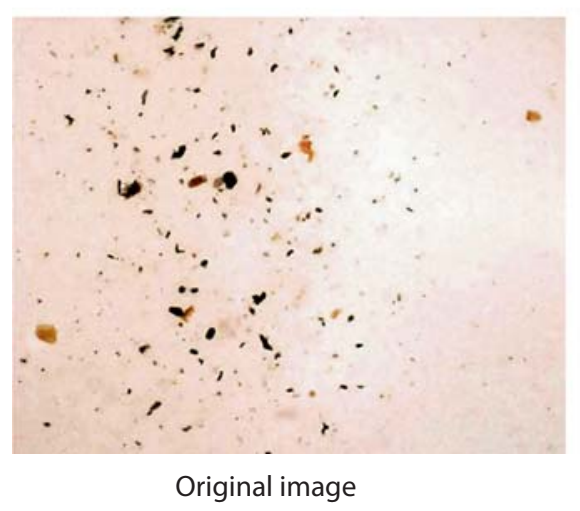

2

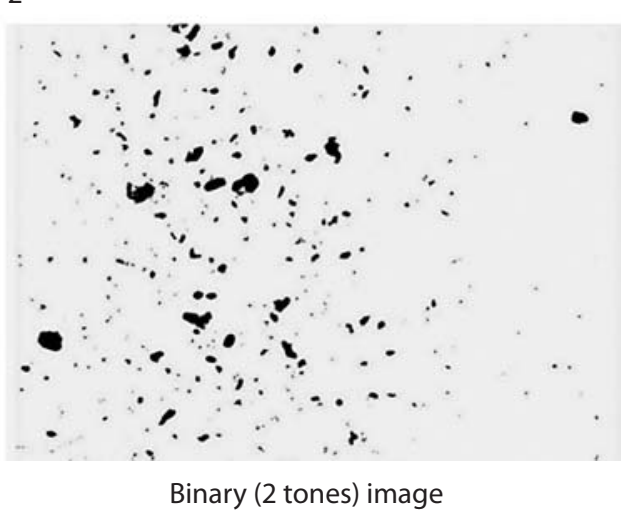

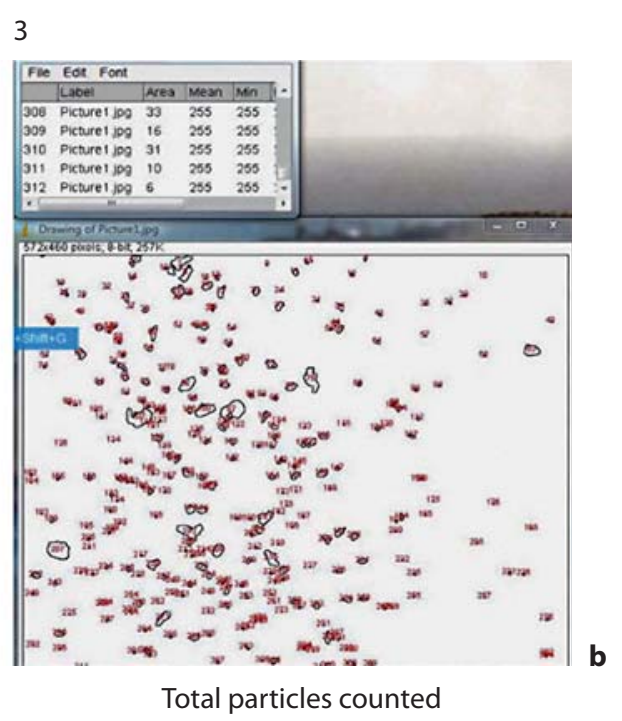

Fig. 1. a Numbered diagram of the halogen immunoassay, adopted from that of Green et al. [35], with the modification that the air sample is collected on the adhesive tape. b Numbered diagram with the sequence of images obtained when counting the total particulates. b1 is the original image, and b2 is the image converted to a binary image (2-tone) with ImageJ, in which the color of all particles present on the image was changed to only black and the background is all white to facilitate the total particle count by the software. After the smallest and largest pixel area and circularity ranges were set, the total particles were counted with ImageJ as is shown in b3. Notice that the particles in all 3 images are in the same location. 
The adhesive tapes containing the air sample were sealed against a 1-cm square (1 for each serum) of mixed-cellulose protein-binding membrane (MCE-PBM) (Millipore Corp., Billerica, Mass., USA) and air bubbles were removed to seal the membrane. Each membrane covered 4 traces (impacts) of the air sample captured on the tape. The sealed MCE-PBM were transferred, with the tape side facing upwards, into the wells (1 membrane per well) of a 12-plate tissue culture Falcon plate (Becton Dickinson) containing $800 \mu \mathrm{l}$ of borate buffer. The membranes were left overnight at $4^{\circ} \mathrm{C}$ to elute allergens onto the surroundings of their particles of origin and to bind to the MCE-PBM-tape sandwich. To remove the buffer, the membranes were washed 3 times (for $10 \mathrm{~min}$ at each washing) with $800 \mu$ l phosphate-buffered saline (PBS) with $0.05 \%$ Tween 20 (Fisher Scientific) on a shaker (all washing steps followed this same protocol). The extraction with borate buffer was followed by the addition of $400 \mu \mathrm{l}$ of $5 \%$ skim milk/PBS $/ 0.05 \%$ Tween 20 to each well and this was left on the shaker for $45 \mathrm{~min}$ at room temperature. This step prevented the nonspecific binding of serum IgE and secondary conjugated antibodies by blocking areas of the membrane in which there was no antigen. The membranes were again washed for 10 min 3 times before the addition of $400 \mu \mathrm{l}$ of human serum (diluted 1:3 in 2\% skim milk/PBS/ Tween 20) to the corresponding wells and were allowed to react overnight at room temperature. The membranes were washed 3 times for $10 \mathrm{~min}$, and $400 \mu \mathrm{l}$ of phosphatase-conjugated goat anti-human IgE (Kierkegaard and Perry Laboratories, Gaithersburg, Md., USA) diluted 1:500 in 2\% skim milk/PBS/0.05\% Tween 20 was added to each well and incubated for $90 \mathrm{~min}$ on a shaker at room temperature. The unbound conjugated antibody was removed by washing 3 times for $10 \mathrm{~min}$. After the third wash, the membranes were left overnight in $800 \mu \mathrm{lBS} / 0.05 \%$ Tween 20 at $-4^{\circ} \mathrm{C}$. The next day the membranes were washed once before the addition of $400 \mu \mathrm{l}$ of 5-bromo-4-chloro-3-indolyl-phosphase/nitroblue tetrazolium (BCIP/NBT) (Kierkegaard and Perry Laboratories) to each well and placed on the shaker. The reaction was monitored every $10 \mathrm{~min}$ for staining until a purple coloration was observed (approx. within 10-30 min). If after $30 \mathrm{~min}$ there was no purple color, the reaction was stopped.

\section{Image Analysis}

The membranes were examined with a Nikon Eclipse $80 \mathrm{mi}-$ croscope (Nikon Instruments, Huntington, N.Y., USA) to which a Nikon DXM 120 F camera (Nikon Instruments, Inc.) was attached, and this camera was connected to a computer. Because the particulates that the Allergenco MK-3 collected appeared as vertical traces on the tape and only 4 traces were able to be covered by the 1-cm square of MCE-PBM, 4 fields per membrane (1 field for each trace on the membrane) were photographed at a $10 \times$ magnification with ImagePro Plus ${ }^{\circledR}$ version 5.6.2.9 (Media Cybernetics, Inc., Bethesda, Md., USA). The membranes were examined at this magnification to cover a broader field and to avoid obtaining blurred images. The contrast, exposure, and illumination enhancements were performed with Adobe Photoshop CS4 Extended software (Adobe Systems Corporation, San Jose, Calif., USA).

The total particles photographed on each field were calculated as shown in figure $1 \mathrm{~b}$ using image analysis software Image $\mathrm{J}^{\circledR}(\mathrm{Re}$ search Service Branch, National Institute of Mental Health, National Institutes of Health, Bethesda, Md., USA). Because the particles in the images varied in color, each image was segmented and converted to binary images ( 2 tones). With this adjustment, any particle present on the membrane turned black while all of the background acquired a white color. Before proceeding to count the particles, the pixel area (square pixels) was set to cover the smallest (1) and largest $(3,000)$ pixel area range and circularity in the range of $0.00-1.00$. With these modifications, it was easier for the software to count the particles of interest as they were of only 1 color without any interference from the background. After these adjustments, all particles of interest were counted and the result for each image recorded. The total particulates for each subject were the sum of the total of the 4 images corresponding to each membrane, and the percentage haloes was calculated by dividing the total haloes (the sum of the haloes of the 4 images) by the total particulates. The percentage of haloes was used throughout the analysis to normalize for the differences in the concentrations of the total particulates on the membranes.

To confirm and identify the particles with haloes we used Adobe Photoshop CS4 Extended, with the aid of the plug-in BlowUp 2.0.4 (Alien Skin Software, Raleigh, N.C., USA). Haloes were confirmed by inverting the image (fig. $2 \mathrm{~d}$ ). In this manner, the white background, which often interfered with the identification of haloes, was eliminated and true haloes appeared as blurred areas of light blue. For particle identification, regions of the noninverted images with haloes were enlarged by $400 \%$ (from a $10 \times$ magnification to a $40 \times$ magnification) with the plug-in mentioned above in order to increase the size of the particle, allowing a better resolution with minimal loss of pixel detail (fig. 2e). For particles hidden by the haloes, edges were highlighted as shown in figure $2 \mathrm{f}$. In this manner, the halo disappeared and only the edge of the particle(s) was visible.

The fungal particles with haloes were grouped as basidiospores, ascospores, hyphae/hyphal fragments (germinating spores or free fragments), deuteromycetes or Fungi Imperfecti (e.g. Fusarium spp., Alternaria spp., Cladosporium spp., and Epicoccum spp.), and unidentified (unID; any haloed particle not able to be identified or classified into any of the previous groups). Each haloed particle was identified and counted on each image based on the following criteria: for basidiospores, being globose, elliptic, or asymmetrical, usually having a $<4$ :1 length/width ratio, an offcenter hilar appendage, sometimes the presence of an apical germ pore, and no branch, scar, or septum; for ascospores, symmetry, no scar, usually having a $>4: 1$ length/width ratio, and often being in groups of 8; for hyphae/fragment, germinating spores or free fungal fragments; for deuteromycetes, based on their characteristic mitospores as described by Smith [41], and for the unidentified group, any haloed particle not able to be classified into any of the previous groups.

\section{Statistical Analysis}

The statistical analyses were performed using Minitab Statistical Software version 15.1.30 (Minitab, Inc., State College, Pa., USA). Because most of the parameters included were not normally distributed, they were expressed as median values. Box plot graphs show the median (horizontal line within the box), the interquantile ranges (box's edges), the ranges of all values within interquantile 1.5 , and any outlier value of the graphed data. A 2 -proportion test was used to compare the percentage of subjects that reacted to each particulate category when grouped by disease, SPT reactivity, or SPT positive extracts. The nonparametrical Mann-Whitney $U$ test was used to compare the percentages of haloes according to the particulate category and when subjects 


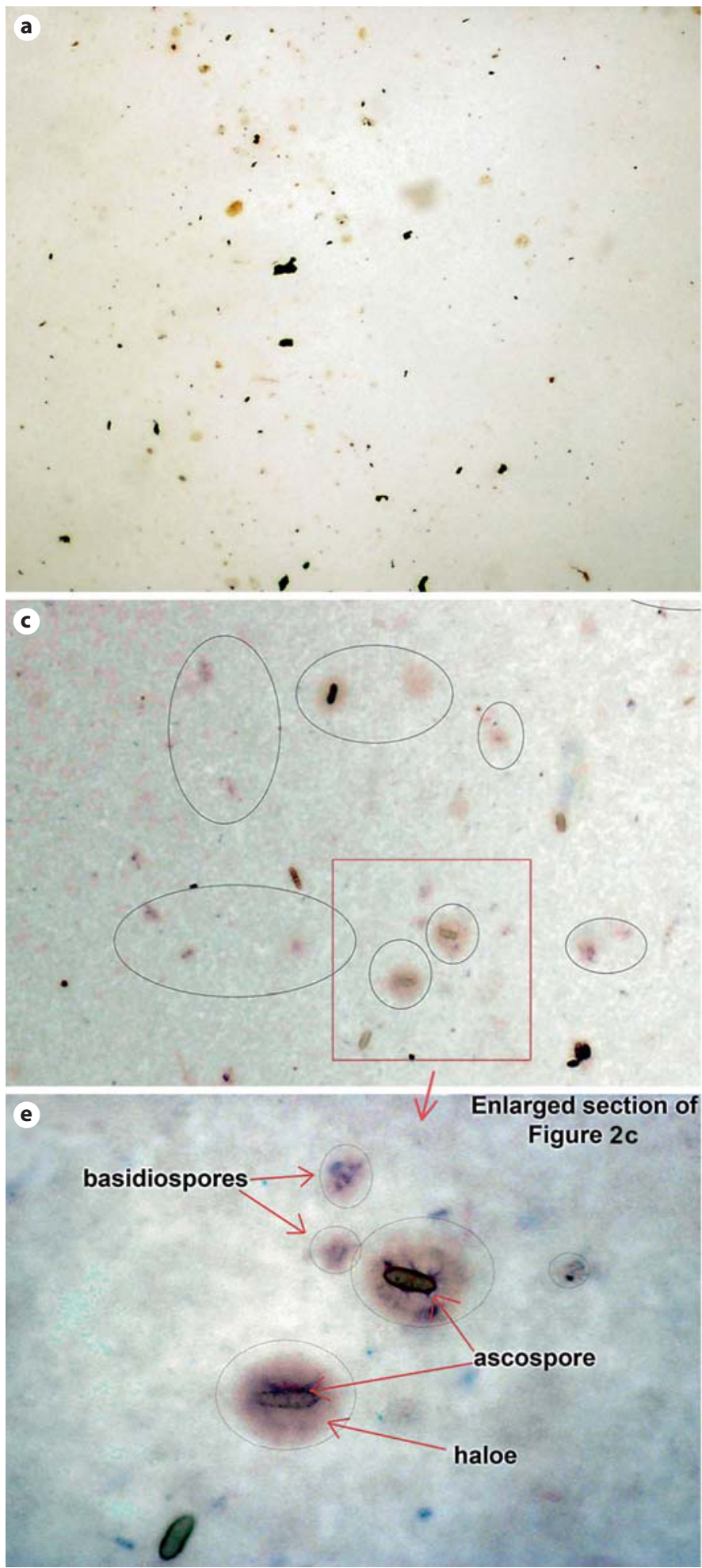

Fig. 2. a Halogen assay image with negative results (no haloes). b Halogen assay image with positive results. c Image with haloed particles serving as an example to demonstrate the 3 image adjustments used to confirm and identify the reactive particles.

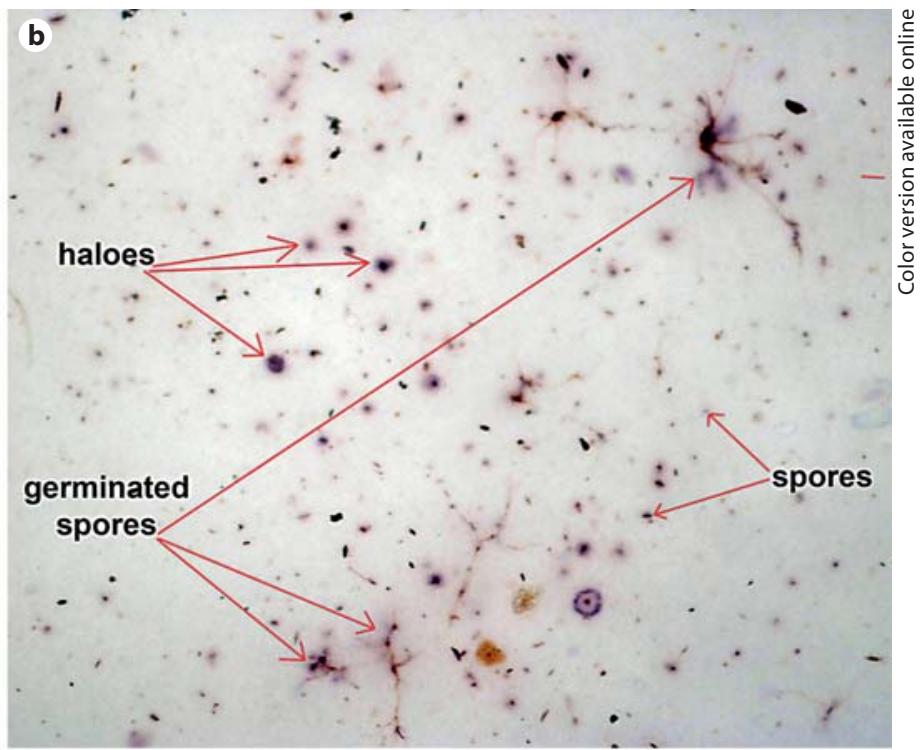

d

Inverted image of Figure 2c
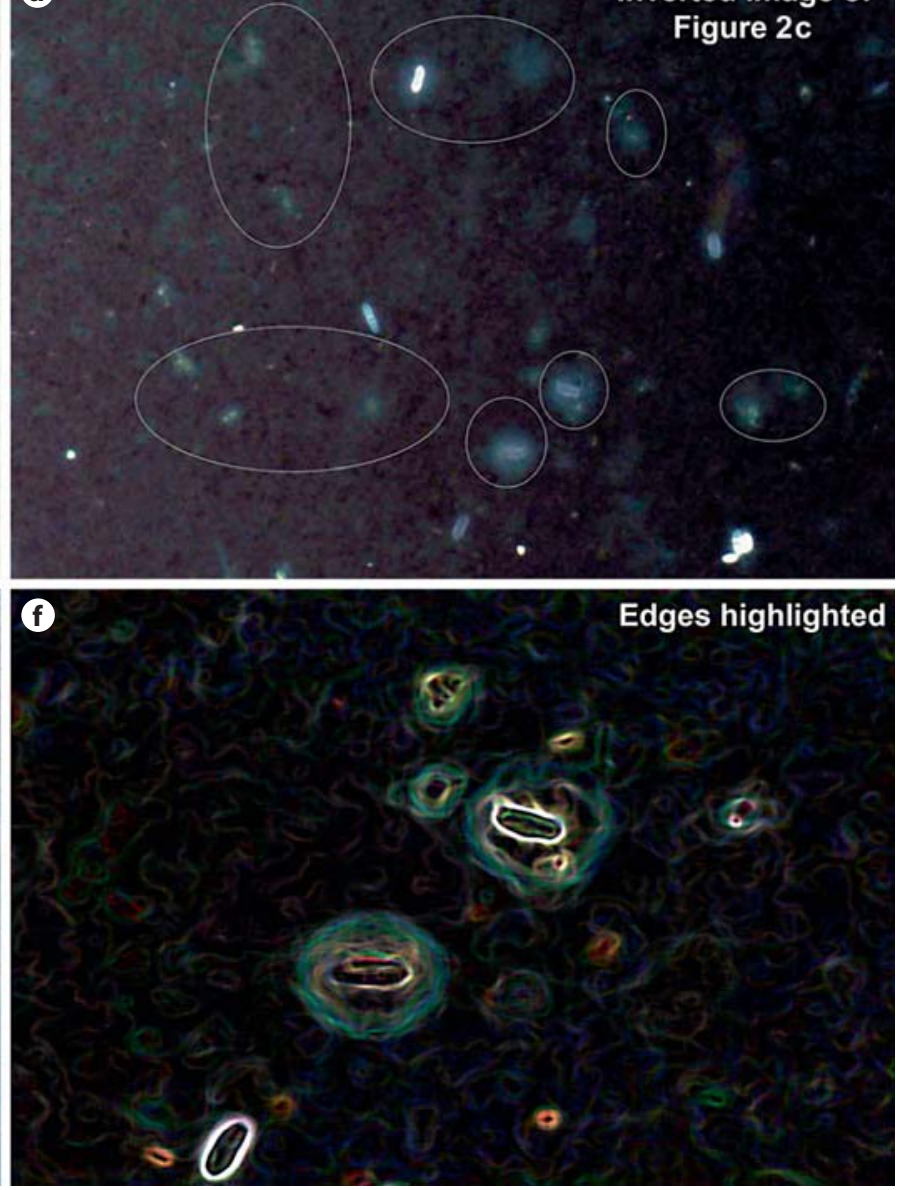

d Inverted image. e Sections of the image enlarged $400 \%$ with Adobe Photoshop with the Blow-up plug-in. f Highlighted edges of the particles that were hidden by the halo. 


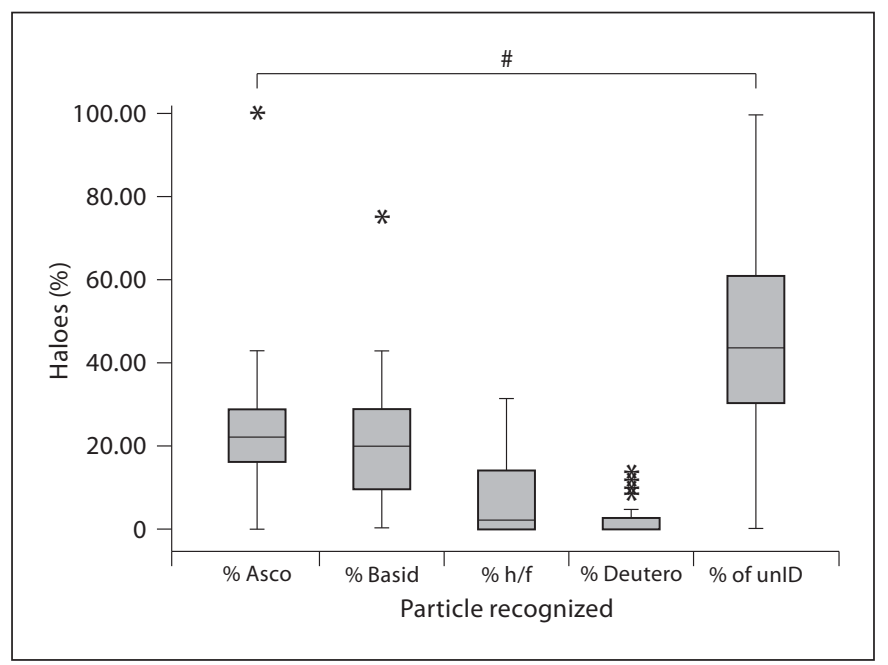

Fig. 3. Box plot of the percentages of individual particles with haloes. Asco = Ascospores; basid = basidiospores; $\mathrm{h} / \mathrm{f}=$ hyphae and fragments; deutero $=$ deuteromycetes. ${ }^{*}$ Outlier; ${ }^{*} \mathrm{p}<0.05$.

were group by disease, SPT allergen source, and number of SPT extracts to which the subjects were positive. The Pearson productmoment correlation coefficient and regression analysis were used to find any correlations between predictors (concentrations of total particles, condition, number of positive extracts, and IgE levels) and the percentages of haloed particles. Each respiratory condition was number-identified according to the International Classification of Diseases developed by the World Health Organization (ICD9): nonallergic rhinitis (472), allergic rhinitis (477), and asthma (493). $\mathrm{p}<0.05$ was considered statistically significant.

\section{Results}

For each patient, the serum was challenged against an outdoor air sample via the halogen immunoassay. Table 1 reports the test subjects, including their condition (asthma, allergic rhinitis, or nonallergic rhinitis), SPT results, and IgE levels, as well as the 2 control subjects. Diagrams of the halogen immunoassay and image analysis to count the total particulates are shown in figure 1; figure 2 shows examples of negative (fig. 2a) and positive (fig. 2b) membranes, an inverted image to confirm a halo (fig. 2d), an enlarged section of a positive membrane (fig. 2e), and the highlighted edges of reactive particles (fig. 2f). Of all haloed particles, 25\% were ascospores, $20 \%$ basidiospores, $7 \%$ hyphae and fungal fragments, $2 \%$ mitospores, and $46 \%$ unID (fig. 3). Of all subjects, $94 \%$ $(\mathrm{n}=31)$ reacted to ascospores, $88 \%(\mathrm{n}=29)$ to basidiospores, $58 \%(\mathrm{n}=19)$ to hyphae/fungal fragments, and
$36 \%(n=12)$ to mitospores (fig. $4 a)$. Significant differences were found with a 2-proportion test when subjects that were positive to basidiospores and ascospores were compared to those who were positive to hyphae/fragments and deuteromycetes. When subjects were grouped by disease (fig. 4b), by SPT positive to a specific allergen source (fig. 4c), or by the number of extracts positive in the SPT (fig. 4d), similar patterns were observed. A significant difference was found in the percentages of haloes to hyphae/fragments when subjects who reacted to 3 or more SPT extracts were compared to those who were positive to 1 extract (fig. $4 \mathrm{~d}$ ).

The percentages of haloed particles among subjects with asthma, allergic rhinitis, or nonallergic rhinitis were determined and a Mann-Whitney U test performed to find statistically significant differences between the groups. Figure 5a shows the median percentages of the haloes for the 3 groups, in which $1.09,4.82$, and $0.39 \%$ corresponded to subjects with asthma, allergic rhinitis, and nonallergic rhinitis, respectively. There was a significant difference between subjects with allergic rhinitis and those with nonallergic rhinitis. There was no significant difference, however, between groups with asthma and nonallergic rhinitis or those with asthma and allergic rhinitis.

The percentages according to the number of positive extracts $(0,1,2$, or 3 or more) and according to positivity to a particular SPT-allergen source (basidiomycetes, deuteromycetes, pollen, or animal allergen) were examined. As shown in figure $5 \mathrm{~b}$, the percentages of haloes were $5.24,1.09,1.61$, and $0.57 \%$ if the participant was SPT positive to 3 or more, 2 or 1 extract or negative to all extracts. There were significant differences between subjects positive to 3 or more extracts and the remaining groups. When subjects were grouped by SPT allergen source, the percentage of reaction was greatest for subjects who were SPT positive to basidiomycetes (4.74\%) as compared to pollen (4.15\%), animal allergens (3.63\%), and deuteromycetes $(3.31 \%)$ as shown in figure $5 c$, but there were no significant differences between the groups.

To determine whether there was any relationship between the percentages of haloes and predictors, Pearson product-moment correlation coefficients were determined. As shown in table 2a, there seem to be correlations, although not very strong ones, between the total haloes and the number of positive SPT extracts and IgE levels. A linear regression analysis (table $2 \mathrm{~b}$ ) was performed between these 2 predictors, and the number of positive extracts was the most significant. Table $2 c$ shows that there was no correlation between each predictor and the percentages of each group of haloed particles. 


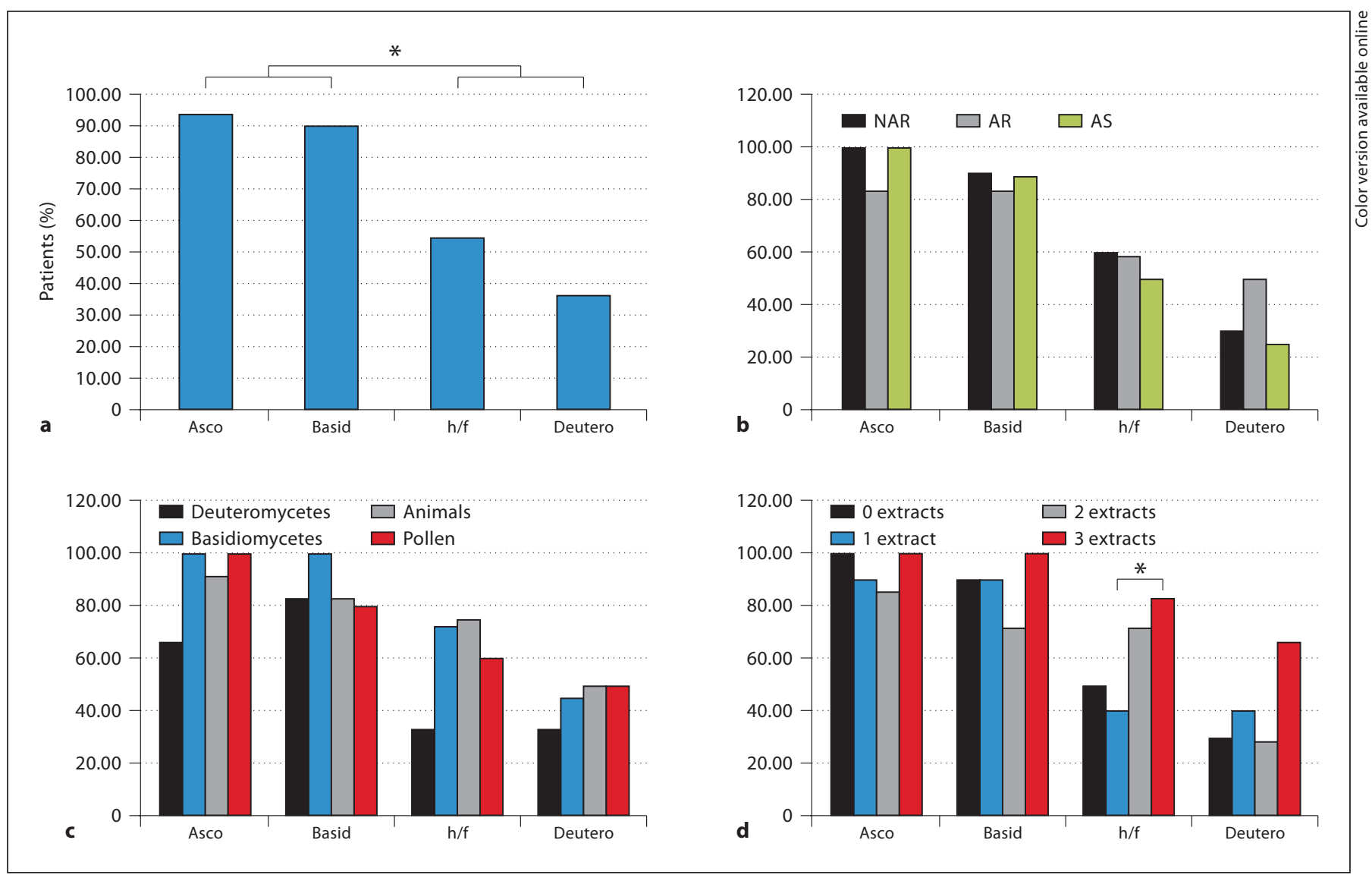

Fig. 4. Percentages of patients that reacted against fungal particles. $\mathbf{a}$ All patients. $\mathbf{b}$ According to disease. NAR = Nonallergic rhinitis; AR = allergic rhinitis; AS = asthma. $\mathbf{c}$ According to allergen group. $\mathbf{d}$ According to positive extracts. Asco = Ascospores; basid = basidiospores; $\mathrm{h} / \mathrm{f}=$ hyphae and fragments; deutero = deuteromycetes. * $\mathrm{p}<0.05$.

\section{Discussion}

Fungal spores are the biological airborne agent most predominant in many areas $[12,23,24,29,42-44]$, and in others fungal fragments have been demonstrated to be reactive in subjects with allergies [19-21]. A significant number of them are small enough to penetrate into the upper and lower airways and induce immunological reactions in subjects with rhinitis and asthma [17]. However, it has been difficult to provide direct evidence that these particles could react with the immune system of individuals with allergic respiratory diseases because extracts are not available for many fungi or fungal fragments. Using whole-air extracts would be nonspecific and may yield secondary reactions. For these reasons, the halogen immunoassay was used to directly test the reactivity of sera from subjects with different types of sensi- tizations and allergic respiratory diseases in relation to airborne allergens.

Particles that were not able to be identified had the highest percentage of haloes, but this category alone was not above 50\%. Particles of fungal origin (ascospores, basidiospores, hyphae/fragment, and deuteromycetes) together accounted for more than $50 \%$ of all halo reactions. The origin of the unID particles could be any organic source with aerodynamic and allergenic attributes. Its identification might be possible with modifications to the halogen immunoassay (e.g. more sensitive image acquisition hardware and/or software, a higher magnification, and more optically sensitive adhesive film). Nevertheless, fungal spores that predominate in the SJ atmosphere, such as basidiospores and ascospores, reacted with the subjects' sera at a higher percentage than mitosporic fungi (fig. 3, 4a). In addition, the same pattern was observed 
Table 2. Pearson product-moment correlation coefficients and linear regression of the percentages of haloes with predictors

a Pearson's correlation between the percentages of haloes and the predictors

\begin{tabular}{ll}
\hline & Percentage of haloes \\
\hline Total particulate & \\
$\quad$ Pearson's correlation coefficient & -0.386 \\
p value & 0.026 \\
Condition & \\
$\quad$ Pearson's correlation coefficient & 0.103 \\
$\quad$ p value & 0.581 \\
Number of positive extracts & \\
$\quad$ Pearson's correlation coefficient & 0.649 \\
$\quad$ p value & 0.000 \\
Total IgE & 0.554 \\
$\quad$ Pearson's correlation coefficient & 0.002 \\
$\quad$ p value & \\
\hline
\end{tabular}

b Linear regression of the percentages of haloes with IgE levels and positive extracts

\begin{tabular}{lllll}
\hline & Constant & Condition & $\begin{array}{l}\text { IgE } \\
\text { levels }\end{array}$ & $\begin{array}{l}\text { Positive } \\
\text { extracts }\end{array}$ \\
\hline t value & 0.0310 & -0.270 & 1.060 & 2.280 \\
p value & 0.763 & 0.790 & 0.220 & 0.031 \\
\hline
\end{tabular}

c Pearson's correlation between percentages of particles with haloes and predictors

\begin{tabular}{lccc}
\hline & Disease & Extracts & IgE levels \\
\hline unID & 0.059 & -0.093 & -0.057 \\
& 0.754 & 0.608 & 0.768 \\
\hline Asco & 0.000 & 0.084 & -0.059 \\
& 1.000 & 0.643 & 0.761 \\
\hline Basidio & -0.060 & 0.031 & 0.129 \\
& 0.750 & 0.865 & 0.506 \\
H/F & 0.015 & 0.037 & -0.036 \\
& 0.934 & 0.836 & 0.855 \\
\hline Deutero & -0.036 & 0.042 & 0.093 \\
& 0.847 & 0.819 & 0.630 \\
\hline
\end{tabular}

Asco = Ascospores; basidio = basidiospores $\mathrm{H} / \mathrm{F}=$ hyphae and fragments; deutero $=$ deuteromycetes. $\mathrm{p}<0.05$ was considered significant.
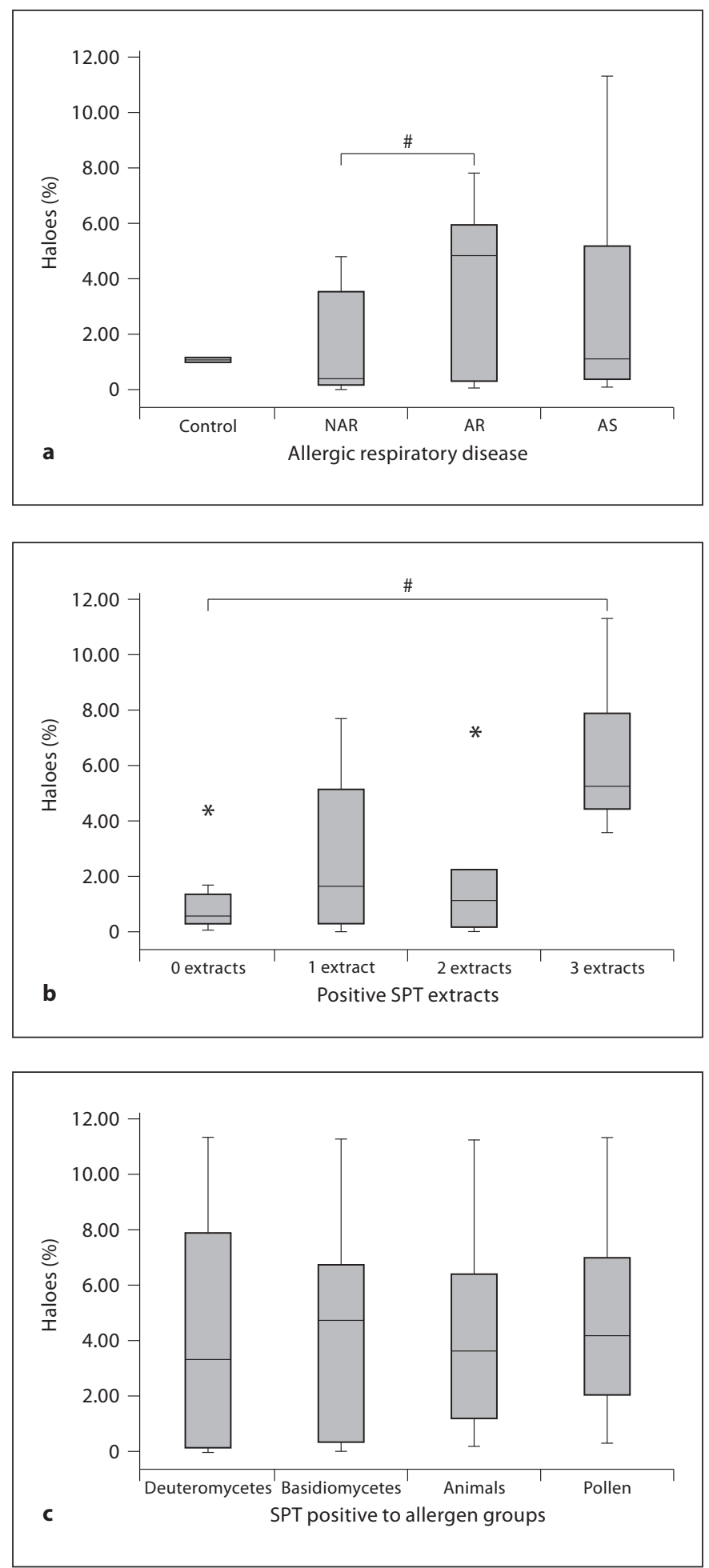

Fig. 5. a Box plot of the percentages of haloes according to respiratory disease. $\mathrm{NAR}=$ Nonallergic rhinitis; $\mathrm{AR}=$ allergic rhinitis; $\mathrm{AS}=$ asthma. $\mathbf{b}$ Box plot of the percentages of haloes according to the number of positive extracts. c Box plot of the percentages of haloes if positive to a particular allergen group in the SPT. * Outlier; ${ }^{\#} \mathrm{p}<0.05$. 
in the 4 scenarios presented in figure 4 when subjects were grouped by disease (fig. 4b) and SPT results (fig. 4c, d). These findings suggest that these spores, which have many attributes causing them to be considered allergenic [44], could be active inducers of exacerbations of rhinitis and asthma during seasons in which their concentrations are very high. Nevertheless, epidemiological and clinical studies must be performed to fully support their active role in respiratory allergies. However, in figure $4 \mathrm{~d}$ an interesting overlap was observed because subjects who were reactive to 3 or more SPT extracts had a higher percentage of haloes to hyphae/fragments and deuteromycetes, although this was not significant in the latter group. A possible explanation might be that the airborne fragment and mitosporic fungi to which they were reactive in the halogen assay may have allergens similar to those of the SPT extracts to which they were positive. This scenario should be further studied because a previous report by Green et al. [35] in which sensitization was examined for mitosporic fungi by different methods found a correlation between halogen assay and allergen-specific IgE levels, but no correlation was found with the SPT.

It seems that for higher degrees of hypersensitivity to airborne allergens, subjects must have high levels of $\operatorname{IgE}$ or multiple-allergen sensitization. This is supported by the fact that patients with allergic-rhinitis, positive SPT reactivity to 3 or more extracts, or high levels of serum IgE had the highest percentages of haloes. These findings are in accordance with various studies in which subjects with atopy and patients suffering from allergic respiratory diseases seemed to react more than nonatopic patients to fungal allergens $[23,35,38]$. Nevertheless, it is feasible that those suffering from nonallergic rhinitis may be also affected by airborne allergens resulting from local sensitization or the expression of immunoglobulin-free light chains that may not be detected by the SPT or assays using IgE, which test for systemic sensitization [46, 47]. Although there are different classifications of nonallergic rhinitis [48], studies have demonstrated that in some subjects with diagnosed nonallergic rhinitis in whom the SPT was negative, nasal challenges show sensitization.

Asthma subjects in this study had lower median percentages of haloes compared to allergic rhinitis subjects and a higher median percentage of haloes (though this was statistically insignificant) compared to nonallergic rhinitis subjects. A lack of sensitization to fungi or low serum levels of IgE could not explain these results because these subjects had high IgE levels and various positive extracts, including fungi. A possible explanation for this finding could be the sample size because the percent- ages of haloes among this group of subjects were not parametrically distributed and some of them demonstrated a higher percentage compared to allergic rhinitis subjects. Also, their serum IgE could be specific for allergens other than those captured at the time of the air sample collection. We must keep in mind that subjects were exposed to somewhat different levels of airborne particulates because air samples were collected on different days. Although it may be interesting to examine the sera air samples collected on the same days, it is extremely difficult to duplicate an air sample unless multiple samplers have the same location and operate at the same time.

Subjects seem to react to airborne fungal allergens in a specific manner not dependent on the total amount of particulate as there was no correlation between the total particulates and the percentage of haloed (total or individually) particles. This may suggest that subjects with asthma and rhinitis experience exacerbations on days when the diversity of the fungal air spores shifts to a particular allergen(s). There are few reports related to fungal spores or to allergenic sensitivity to these particulates in tropical climates although ascospores and basidiospores seem to be the most prevalent outdoor fungal spores in tropical environments [14-16]. For this reason, epidemiological studies are needed to confirm that susceptible individuals may experience episodes of asthma and rhinitis when these groups of spores are in very high concentrations in the atmosphere.

One interesting observation was that sera from more than half of the subjects were also reactive to hyphae and hyphal fragments (fig. 4a). Few studies have described these as possible allergens [17-21] even though most of the described fungal allergens in the literature originate from induced systems expressed when a spore is metabolically active and germinating or when the hyphae is elongating [24]. As previously described by Green et al. [21], fungal fragments can retain allergens when airborne and participate as possible inducers of allergic respiratory diseases. Furthermore, it is important to mention that they have been previously described as biocontaminants [18] and, as stated by Cho et al. [17], they have aerodynamic properties, can become airborne indoors and outdoors, and deposit in the respiratory mucosa. Aerodynamically, one may think that they might not reach the lower airways because of their length. However, it is possible that submicron fungal fragments are released and suspended in the air and reach the upper and lower respiratory tract. Finally, the widths of many fungal fragments do not reach $5 \mu \mathrm{m}$, increasing the possibility of reaching the lower respiratory tract. 
Although the percentages of haloes were low, it should be taken into consideration that, for technical reasons, only an area was analyzed instead of the whole membrane. It was almost impractical to photograph the membrane at a lower resolution for a broader field or to acquire images from the complete MCE-PBM with higher magnifications. The former would have yielded images with little detail of the reacting particles while with the latter would have produced lots of images and time limitations would have prevented analysis of these data. Time limitations also supported our decision to use image analysis (i.e. ImageJ, Adobe Photoshop ${ }^{\circledR}$ CS4 Extended, and the Blow-up ${ }^{\circledR}$ plug-in) to automatically count the total particles in each image, taking a percentage of the sample and identifying only the reacting particles, and to identify the reactive particulates. It must be borne in mind, however, that humans take in approximately 10,000 liters of air per day [49]. An extrapolation of the results from the sample analyzed in this study's assay indicates that the amount of fungal particulates that a susceptible individual breathes daily could be significant.

Another limitation of this study was the size of the sample studied and the controls included. We acknowledge that this may have had an impact on the strength of the statistical analysis and the interpretation of the results. However, with the limited number of subjects studied, differences in reactivity to different fungal particles were observed, and in some cases these were statistically significant. Furthermore, in previous studies in which the halogen immunoassay has been used, researchers have captured particles directly on the membrane, as was described for cat and fungal allergens $[19,35,36,38,40]$. In this study, the air samples had to be collected on the sticky side of the tape. Initially, we conducted air sampling and particles did not adhere to the surface of the membrane. While collecting air samples, the particles bounced as the surface did not have an adhesive side. Although it may have prevented the germination of fungal spores, germinating spores and fungal fragments were observed as seen in figure $2 \mathrm{~b}$ although they did not correspond to the fungal particles with highest percentage of haloes. Finally, one may question the reliability of the identification of the particles, as the magnification used (only 10×) may have been a limitation for the detection of small fungal spores. This magnification was used to obtain a larger field and to limit the images analyzed. Although small spores may have been missed, the Blow-up plug-in worked well in enlarging. As mentioned in Materials and Methods and illustrated in figure $2 \mathrm{f}$, for identification purposes (whenever possible as not all particles were able to be identified and were thus classified as unID) the edges of the reacting particle were highlighted allowing the visualization of its symmetry (if any); based on the criteria mentioned previously, the particle was then classified as either an ascospore, a basidiospore, a fragment/germinating spore, a mitospore, or unID. In addition, the sections with haloes of the images were enlarged nearly $400 \%$ without damaging pixel details; this allowed us to detect small hyaline basidiospores as shown in figure 2e. Interestingly, we did not observe pollen or any identifiable pollen particulates in any of the sealed membranes. This does not necessarily rule out that pollen did not react with the sera as submicroscopic grains and that small fungal spores such as those of Aspergillus/ Penicillium may have been missed.

It is also important to discuss the fact that monoclonal antibodies have been used in previous studies to detect levels of sensitization to other allergens, including those cases in which halogen and other immunological assays have been used $[19,36,38,40]$. These antibodies allowed the detection and quantification of sensitization to specific and characterized allergens; however, this is not possible with many airborne allergens, including fungal spores and fragments, as there are only monoclonal antibodies to few fungi. In the case of basidiomycetes, allergens have been fully described in just a few cases $[32,33]$ and, to our knowledge, there are no published studies of monoclonal antibodies to basidiomycete allergens.

It could also be questioned that the sera in this study reacted to carbohydrate instead of protein determinants of airborne fungal spores. There have been numerous studies with different types of allergen sources in which the significance of carbohydrate determinants has been studied [11, 50-53]. Several of these investigations have concluded that these epitopes participate in the crossreactivity between allergen sources, while in other cases they have been useful in the characterization of certain proteins. In our case, we cannot disregard this possibility as studies of carbohydrate determinants in basidiomycetes have not been carried out. In addition, we do not think that cross-reactivity has occurred between mitosporic fungi or basidiomycetes or between these groups and basidiomycete or ascomycete allergens because this phenomenon has not been described to in this scenario. This possibility is worth being studied as it has been reported that cross-reactivity is more common than previously thought in fungi. Finally, we should point out that the controls showed some reactivity to the fungal particulates. Fungi can produce alkaline phosphatase, and some can do this in a constitutive manner [54-56]; how- 
ever, although this is not shown, we did perform control tests excluding human serum, the conjugate, or the substrate, and no purple color characteristic of a halo was observed.

In summary, we described the allergenic potential of the airborne fungal particulate that predominates in the atmosphere of SJ in individuals suffering from allergic respiratory diseases. To our knowledge, this is the first time in which it has been documented that airborne basidiospores and ascospores, which predominate in our setting, were directly tested with a pool of subjects and were demonstrated to be reactive to the immune systems of subjects with allergic respiratory diseases. This work provides additional evidence of the possible significance of outdoor airborne fungal allergens and their effect on the susceptible population, and it could lead to further investigation with respect to the participation of basidiomycetes and ascomycetes in allergic respiratory diseases.

\section{Acknowledgements}

We would like to acknowledge the Associate Deanship of Biomedical Affairs of the School of Medicine of the UPR-MSC for their financial support of this project. We thank Merck Sharp and Dome (I.A.) Co. for the support to cover the costs of this publication. In addition, we are very grateful for the subjects' participation. We thank Joenice González-De León (graduate student) for her technical support, Dra. Edna Aquino and Mrs. Petraleigh Pantoja for their contributions as medical technicians, and Borinquen Laboratories (Caguas, Puerto Rico) and the laboratory of Menonita Hospital (Cayey, Puerto Rico) for their support with extracting the sera from the blood samples and the determination of IgE levels. Also, we gratefully acknowledge the excellent editing work of Dr. Mary H. Mays. This study was supported by the UPR School of Medicine Endowed Health Services Research Center Grants 5S21MD000242 and 5S21MD000138, from the National Institutes of Health. Its contents are the sole responsibility of the authors and do not necessarily represent the official views of NIH.

\section{References}

1 Platts-Mills TA, et al: Determinants of clinical allergic disease: the relevance of indoor allergens to the increase in asthma. Am J Respir Crit Care Med 2000;162:S128-S133.

$\checkmark 2$ Platts-Mills TA, Rakes G, Heymann PW: The relevance of allergen exposure to the development of asthma in childhood. J Allergy Clin Immunol 2000;105:S503-S508

3 World Health Organization: World Health Statistics 2006 (WHOSIS). Geneva, World Health Organization, 2010.

4 Perez-Perdomo R, et al: Prevalence and correlates of asthma in the Puerto Rican population: Behavioral Risk Factor Surveillance System, 2000. J Asthma 2003;40:465-474.

5 Perez-Perdomo R, et al: The prevalence of asthma and the utilization of medical services among those insured by a health-services company in Puerto Rico, 1996-1997 (in Spanish). Bol Asoc Med PR, 1999;91:91-97.

-6 Montealegre F, et al: Age, gender and seasonal patterns of asthma in emergency departments of southern Puerto Rico. PR Health Sci J 2002;21:207-212.

-7 Montealegre F, Chardon D, Tarrats H: Environmental factors precipitating bronchial asthma exacerbations in southern Puerto Rico: a pilot study. J Asthma 1993;30:219227.

8 Cohen RT, et al: Health-care use among Puerto Rican and African-American children with asthma. Chest 2006;130:463-471. $\checkmark 9$ Seguinot-Medina S, Rivera-Rentas A: Risk assessment and community participation model for environmental asthma management in an elementary public school: a case study in Puerto Rico. Int J Environ Res Public Health 2006;3:76-85.

10 Loyo-Berrios NI, et al: Air pollution sources and childhood asthma attacks in Catano Puerto Rico. Am J Epidemiol 2007; 165:927935.

-11 Kumar A, Kurup VP: Murine monoclonal antibodies to glycoprotein antigens of Aspergillus fumigatus show cross-reactivity with other fungi. Allergy Proc 1993;14:189-193.

12 Kurup VP, Shen HD, Banerjee B: Respiratory fungal allergy. Microbes Infect 2000;2:11011110

13 Li DW, Kendrick B: A year-round study on functional relationships of airborne fungi with meteorological factors. Int J Biometeorol 1995;39:74-80.

14 Quintero E, Rivera-Mariani F, BolañosRosero B: Analysis of environmental factors and their effects on fungal spores in the atmosphere of a tropical urban area (San Juan, Puerto Rico). Aerobiologia 2009;26:113-124.

15 James TY, Vilgalys R: Abundance and diversity of Schizophyllum commune spore clouds in the Caribbean detected by selective sampling. Mol Ecol 2001;10:471-479.

16 Chew FT, et al: Evaluation of the allergenicity of tropical pollen and airborne spores in Singapore. Allergy 2000;55:340-347.
17 Cho SH, et al: Aerodynamic characteristics and respiratory deposition of fungal fragments. Atmos Environ 2005;39:5454-5465.

18 Gorny RL, et al: Fungal fragments as indoor air biocontaminants. Appl Environ Microbiol 2002;68:3522-3531.

19 Green BJ, Schmechel D, Tovey ER: Detection of aerosolized Alternaria alternata conidia, hyphae, and fragments by using a novel double-immunostaining technique. Clin Diagn Lab Immunol 2005;12:1114-1116.

20 Green BJ, Sercombe JK, Tovey ER: Fungal fragments and undocumented conidia function as new aeroallergen sources. J Allergy Clin Immunol 2005;115:1043-1048.

21 Green BJ, et al: Airborne fungal fragments and allergenicity. Med Mycol 2006;44(suppl 1):S245-S255.

22 Dales RE, et al: Influence of ambient fungal spores on emergency visits for asthma to a regional children's hospital. Am J Respir Crit Care Med 2000;162:2087-2090.

23 Simon-Nobbe B, et al: The spectrum of fungal allergy. Int Arch Allergy Immunol, 2008; 145:58-86.

24 Horner WE, et al: Fungal allergens. Clin Microbiol Rev 1995;8:161-179.

25 Brander KA, Pichler WJ, Helbling A: Isolation of basidiomycete allergens. Int Arch Allergy Immunol 1999;118:302-303.

26 Helbling A, et al: Allergy to basidiomycetes. Chem Immunol 2002;81:28-47. 
27 Denning DW: The link between fungi and severe asthma: a summary of the evidence. Eur Respir J 2006;27:615-626.

28 Black PN, Udy AA, Brodie SM: Sensitivity to fungal allergens is a risk factor for lifethreatening asthma. Allergy 2000;55:501504.

29 Levetin E, Horner WE: Fungal aerobiology: exposure and measurement. Chem Immunol 2002;81:10-27.

- 30 Levetin E, Van de Water P: Environmental contributions to allergic disease. Curr Allergy Asthma Rep 2001;1:506-514.

31 Bartra-Tomas J: Mesa redonda: estado actual de la alergia a hongos. Allergy Clin Immunol 2003;107(suppl 3)430-440.

- 32 Horner WE, et al: Basidiomycete allergy: identification and characterization of an important allergen from Calvatia cyathiformis. Int Arch Allergy Appl Immunol 1991;94: 359-361.

-33 Horner WE, Reese G, Lehrer SB: Identification of the allergen Psi c 2 from the basidiomycete Psilocybe cubensis as a fungal cyclophilin. Int Arch Allergy Immunol 1995; 107:298-300.

34 Doloen WK: Skin testing technique. Immunol Allergy Clin North Am, 2001;21:273279.

35 Green BJ, Yli-Panula E, Tovey ER: Halogen immunoassay, a new method for the detection of sensitization to fungal allergens; comparisons with conventional techniques. Allergol Int 2006;55:131-139.

36 O’Meara TJ, et al: Detection of inhaled cat allergen. Lancet 1998;351:1488-1489.

-37 Tovey ER, et al: New immunodiagnostic system. Aerobiologia 2000;16:113-118.
38 Mitakakis TZ, Barnes C, Tovey ER: Spore germination increases allergen release from Alternaria. J Allergy Clin Immunol 2001; 107:388-390.

39 Green BJ, Mitakakis TZ, Tovey ER: Allergen detection from 11 fungal species before and after germination. J Allergy Clin Immunol 2003;111:285-289.

40 Green BJ, et al: Enumeration and detection of aerosolized Aspergillus fumigatus and Penicillium chrysogenum conidia and hyphae using a novel double immunostaining technique. J Immunol Methods 2005;307: 127-134.

41 Smith EG: Sampling and Identifying Allergenic Pollens and Molds: An Illustrated Identification Manual for Air Samplers. San Antonio, Blewstone Press, 1990.

42 Burge HA: An update on pollen and fungal spore aerobiology. J Allergy Clin Immunol 2002;110:544-552.

43 Burge HA, Rogers CA: Outdoor allergens. Environ Health Perspect 2000;108(suppl 4):653-659.

44 Newson R, et al: Fungal and other spore counts as predictors of admissions for asthma in the Trent region. Occup Environ Med 2000;57:786-792.

45 Gregory P: The Microbiology of the Atmosphere. London, Leonard Hill Books, 1961.

46 Carney AS, et al: Atypical nasal challenges in patients with idiopathic rhinitis: more evidence for the existence of allergy in the absence of atopy? Clin Exp Allergy 2002;32: 1436-1440.

47 James LK, Durham SR: Rhinitis with negative skin tests and absent serum allergenspecific IgE: more evidence for local IgE? J Allergy Clin Immunol 2009;124:1012-1013.

48 Scadding GK: Non-allergic rhinitis: diagnosis and management. Curr Opin Allergy Clin Immunol 2001;1:15-20.
49 Kanda A, et al: Eosinophil-derived IFNgamma induces airway hyperresponsiveness and lung inflammation in the absence of lymphocytes. J Allergy Clin Immunol 2009; 124:573-582, 582 e1-9.

50 Crameri $\mathrm{R}$, et al: Cross-reactivity among fungal allergens: a clinically relevant phenomenon? Mycoses 2009;52:99-106.

51 Malandain H: IgE-reactive carbohydrate epitopes - classification, cross-reactivity, and clinical impact. Eur Ann Allergy Clin Immunol 2005;37:122-128.

52 Shen HD, et al: Cross-reactivity among antigens of different airborne fungi detected by ELISA using five monoclonal antibodies against Penicillium notatum. Zhongahua Yi Zue Za Zhi (Taipei) 1990;46:195-201.

53 Canini A, et al: Localisation of a carbohydrate epitope recognised by human IgE in pollen of Cupressaceae. J Plant Res 2004;117: 147-153.

54 Baakza A, Dave BP, Dube HC: Ferric reductase, superoxide dismutase and alkaline phosphatase activities in siderophore producing fungi. Indian J Exp Biol 2004;42:217219.

55 Guimarães L, et al: Extracellular alkaline phosphatase from the filamentous fungus Aspergillus caespitosus: purification and biochemical characterization. Folia Microbiol (Praha) 2003;48:627-632.

56 Morales AC, et al: Properties of a constitutive alkaline phosphatase from strain $74 \mathrm{~A}$ of the mold Neurospora crassa. Braz J Med Biol Res 2000;33:905-912. 